\title{
Performance of Blind Cooperative Spectrum Sensing under Nonuniform Signal and Noise Powers
}

\author{
Lucas dos Santos Costa, Roberto Cesar Dias Vilela Bomfin, Dayan Adionel Guimarães, \\ and Rausley Adriano Amaral de Souza, Member, IEEE
}

\begin{abstract}
This paper addresses the performance, the report channel traffic and the computational complexity of two blind centralized cooperative spectrum sensing schemes under the effect of unequal and dynamical noise and received signal powers. The first one is the generalized likelihood ratio test, applying decision fusion, and weighted and non-weighted data fusion of samples and eigenvalues. The second one is the circular folding cooperative power spectral density split cancellation method, applying data fusion. It is demonstrated that different configurations of the system parameters may influence the above three metrics in different extents, meaning that none of the techniques is capable of overcoming the others in all metrics simultaneously. As a consequence, the choice of the most suitable technique must be made to match the specific scenario to which the technique is intended to be applied. The results presented in this paper serve as guidelines for assisting this choice.
\end{abstract}

Index Terms-Circular folding cooperative power spectral density split cancellation, cognitive radio, data fusion, decision fusion, eigenvalue fusion, generalized likelihood ratio test, nonuniform noise, spectrum sensing, unequal signal-to-noise ratio, dynamical noise.

\section{INTRODUCTION}

$\mathbf{T}$ HE unprecedented demand for new telecommunication systems and services is currently motivating a huge research effort for developing new technologies and standards, as happens for instance with the fifth generation $(5 \mathrm{G})$ of wireless communication networks, the wireless sensor networks (WSN) and the Internet of things (IoT). The congestion of the radiofrequency spectrum as a possible consequence of the high expected number of new transceivers has become an issue, representing the main bottleneck to the development of such systems and services.

However, it has been verified that a significant portion of frequency bands is in fact underutilized, in spite of the apparent spectrum scarcity problem inherited by the current fixed allocation policy [2]. In this policy, frequency bands

L. S. Costa, D. A. Guimarães, and R. A. A. de Souza are with the National Institute of Telecommunications (Instituto Nacional de Telecomunicações - Inatel), Santa Rita do Sapucaí, MG, Brazil. R. C. D. V. Bomfin is with the Vodafone Chair Mobile Communication Systems, Technische Universität Dresden D-01062, Dresden, Germany. E-mail: \{lucass;dayan;rausley\}@inatel.br, roberto.bomfin@ifn.et.tu-dresden.de.

This work was supported in part by CNPq under Grant 308365/2017-8, and in part by Finep, with resources from Funttel, Grant No. 01.14.0231.00, under the Radiocommunication Reference Center (Centro de Referência em Radiocomunicações - CRR) project of the Inatel.

A shorter conference-type version of this paper was presented in the XXXIV Simpósio Brasileiro de Telecomunicações e Processamento de Sinais (SBrT 2016), Santarém, PA, Brazil, September 1-4, 2016 [1].

Manuscript received April 30, 2017; revised August 30, 2017.

Digital Object Identifier: 10.14209/jcis.2018.17 are permanently and exclusively reserved to the incumbent or primary network.

The cognitive radio (CR) [3] concept has recently emerged as a possible solution to this scarcity problem. CR-enabled secondary user ( $\mathrm{SU}$ ) devices that form the secondary network make use of a flexible spectrum allocation and access strategy in which the primary radio-frequency bands are used opportunistically, in a shared manner with the primary network.

A key enabler of the opportunistic access is the spectrum sensing [4]. It is responsible for detecting unused primary frequency bands (the so-called white-spaces or spectrum holes) that can be occupied by the SU terminals. Thus, the spectrum sensing task must be accurate and fast enough to avoid harmful interferences to the primary user (PU) terminals, yet providing a high data throughput to the secondary network.

If a single SU senses the spectrum, erroneous decisions on the actual occupation state of the channel may be made mainly due to multi-path fading, shadowing and the hidden terminal problem [4]. The accuracy of the spectrum sensing can be improved if more than one SU cooperate to decide on the channel occupation state, thanks to the spatial diversity experienced by SUs located at different positions.

In centralized cooperative spectrum sensing, the binary decisions made by the SUs, or the samples collected by the SUs (or some quantity derived from them) are transmitted to a secondary network element called fusion center (FC), where the final global decision on the channel state is made. When the binary decisions are sent to the $\mathrm{FC}$, a centralized decision fusion cooperative spectrum sensing takes place, i.e., the FC receives the individual binary decisions from all SUs and combine them to make a centralized global decision. On the other hand, when the received signal measurements made by the SUs, or related quantities, are sent to the FC, a centralized data fusion cooperative spectrum sensing is performed. In this case, the FC receives the measurements or related quantities from each SU and process them to form the test statistic that will be used to allow for the global decision on the sensed channel occupation.

\section{A. Related work}

The way in which the PU signal is sensed and processed by an SU determines the specific local spectrum sensing technique. As far as narrowband PU signals are concerned, energy detection [5], [6], matched filter detection [7] and cyclostationary feature detection [8] are widely discussed in the literature. For wideband PU signals, recent studies point 
to three major techniques: energy detection [9], [10], waveletbased detection [11] and compressed (or compressive) sensing detection [12], [13].

Eigenvalue-based detection [14]-[20] is one of the most recent and promising techniques. Likewise energy detection, it can be applied to either narrowband or wideband signals. The test statistic is computed from the eigenvalues of the received signal sample covariance matrix, yielding several different possibilities, for instance the eigenvalue-based generalized likelihood ratio test (GLRT), the maximum-minimum eigenvalue detection (MMED), also known as eigenvalue ratio detection (ERD), and the maximum eigenvalue detection (MED), also named Roy's largest root test (RLRT) [17], [18].

The most important attractiveness of the eigenvalue-based spectrum sensing is that no a priori knowledge about the PU signal is needed for computing the test statistic. In some schemes, like the GLRT and the MMED, the knowledge of the thermal noise variance is not needed as well, meaning that these are considered blind spectrum sensing schemes.

Semi-blind spectrum sensing techniques are those in which the PU signal characteristics are not needed, but the noise variance information is used to compute the test statistic. It is known that the detection performance of such techniques degrades considerably in practice due to the uncertainty in the estimated noise variance. On the other hand, even some blind methods, such as the eigenvalue-based GLRT and the MMED, suffer from performance degradation under nonuniform noise condition, i.e., when noises are assumed to have unequal variances at the inputs of the receivers of the SUs. A generic diagonal noise covariance matrix is allowed in order to take into account these unequal variances or calibration uncertainties at different receiver front-ends. The receiver front-ends in which the noise power is unknown and not assumed to be equal are sometimes referred to as uncalibrated receivers.

Several researchers have proposed techniques to enhance detection performance under nonuniform noise. In [21], robust spectrum sensing methods based on the Gerschgorin disk and the Gerschgorin radii were proposed, but the resultant test statistics do not exhibit the constant false alarm rate (CFAR) property, although this property is claimed by the authors.

In [22] it is proposed a GLRT-based detector for a scenario in which the noise that affects the elements of the multiantenna receiver has different and unknown variances, and for an arbitrary signal-to-noise ratio (SNR). The method requires solving a non-convex optimization problem. All of the detectors formulated in [22] are assumed to have knowledge of the signal rank, which is an unknown parameter when, for instance, it is related to the number of PUs simultaneously transmitting.

In [1] are addressed the performance and the reporting (control) channel traffic in the decision fusion, and in the weighted and non-weighted sample and eigenvalue fusion schemes, under variable noise levels among the SUs, considering the cooperative spectrum sensing with the eigenvaluebased GLRT. Two empirical weighting schemes are proposed to control the combination of the samples or the eigenvalues received at the FC. This is made to confer robustness to the sample and eigenvalue fusion in the case of nonuniform noise, in which the different noise variances were kept unchanged from one sensing interval to the next. The channels between the PU transmitter and the receivers of the SUs are assumed to exhibit flat and slow fading, with identical average SNR for all SUs. Results are presented for a fixed number of SUs, each having a fixed number of antennas.

The performance of the GLRT under nonuniform noise and received signal powers is also analyzed in [23], assuming sensing channels subjected to correlated shadowing. The sensing channels are modeled by a three-dimensional (3D) correlated shadowing model in which the sensors are assumed to be moving uniformly distributed in an 3D space. Similarly to [1], in [23] it is also proposed one weighting scheme for the eigenvalue fusion and one weighting scheme for the sample fusion. However, differently from [1], these schemes were developed based on the maximum likelihood estimate of the noise variance, which provides better performances results when compared to the ones considered in [1].

The authors of [24] address the problem of multi-antenna spectrum sensing, considering the correlation between the received signals at different uncalibrated receivers in the presence of additive white Gaussian noise (AWGN). In order to compute the test statistic, it is proposed to combine the weighted estimated correlations between all antenna pairs. The performance of the proposed detector is optimized by tuning the weighting factors used in the test statistic.

The cooperative power spectral density split cancellation (CPSC) is one of the most recent blind and robust spectrum sensing methods proposed in the literature [25], [26]. The test statistic is constructed in such a way that the noise variance is canceled-out. A modified version of the CPSC named circular folding cooperative power spectral density split cancellation (CF-CPSC) is proposed in [27]. It maintains the low complexity and robustness against nonuniform-dynamical noise (when noise variances are different among the SUs and vary from one sensing interval to the next) with respect to the original CPSC, yielding considerable performance improvements over its previous version.

A large number of other researches related to the influence of nonuniform noise or, more generally, of arbitrary and varying SNRs, on the spectrum sensing performance can be found in the literature. The works cited here are, thus, far from exhaustive.

\section{B. Contributions and structure of the paper}

This paper is an extended and improved version of [1], in which the weighting schemes proposed in [23] are adopted instead of those applied in [1]. The performances of two blind centralized cooperative spectrum sensing schemes under uniform and nonuniform-dynamical signal and noise powers are assessed, considering different numbers of SUs and different numbers of antennas in each SU. The first scheme applies the eigenvalue-based GLRT with decision fusion, and with weighted and non-weighted data fusion of samples and eigenvalues. The second scheme applies the CF-CPSC with total and with partial data fusion. The performances are then traded off against the report channel traffic and the computational complexity of the techniques. 
The following scenarios are considered in the case of the eigenvalue-based GLRT: i) it is analyzed under weighted and non-weighted sample fusion (WSF and SF, respectively), with the eigenvalues of the received signal covariance matrix computed at the FC to form the test statistic; ii) a modified GLRT is analyzed under weighted and non-weighted eigenvalue fusion (WEF and EF, respectively), now with the eigenvalues computed at the SUs and sent to the FC, where the test statistic is formed; iii) the GLRT is applied in each SU equipped with multiple antennas, and local decisions are sent to the FC in a decision fusion (DF) scheme under the combining rules OR, AND and majority-voting (MAJ).

In the case of the CF-CPSC, the following scenarios are addressed: i) total sample fusion (TSF), a case in which the entire processing regarding the computation of the test statistic is performed at the FC; ii) partial sample fusion (PSF), a case in which parts of the test statistic are computed by the SUs to save report channel resources, and the final test statistic is computed at the FC. In this case, the CF-CPSC of [27] is slightly modified to operate with multiple antennas.

To take into account situations of more practical significance, two scenarios regarding the noise variances are considered: i) uniform noise, in which the noise variances are the same in all receivers of the SUs; ii) nonuniform-dynamical noise, in which different and time-varying (from one sensing interval to the next) variances among SUs are considered. Moreover, nonuniform SNRs at the receivers of the SUs are assumed, and the channels between the PU transmitter and these receivers exhibit frequency-selective and slow fading.

In summary, the main novelties with respect to [1] are: i) the inclusion of the CF-CPSC and its modified version (for multiple antennas) in the analyses; ii) the computational complexity analysis of the underlying techniques; iii) the noise variances are allowed to vary from one sensing time to the next; iv) the average SNRs are assumed nonuniform to account for different distances from the PU transmitter to the SU receivers; v) the channels between the PU transmitter and the SU receivers are frequency-selective, what is compatible with the wireless regional area network (WRAN) standard IEEE 802.22 [28], [29]; vi) the number of SUs and antennas per SU are varied to allow for the choice of better system configurations; and vii) the weighting schemes adopt the more accurate noise estimation process suggested in [23], yielding improved performances.

The remainder of the paper is organized as follows: a short background and the system model are given in Section II. Section III describes the approaches adopted for constructing the test statistic of the GLRT and the CF-CPSC, as well as those adopted in the fusion of the spectrum sensing information at the FC. Numerical performance results and discussions are given in Section IV. The trade-off analysis regarding performance, report channel traffic and computational complexity is made in Section V. Section VI concludes the paper and gives some directions for further related research.

\section{BACKGROUND AND SySTEM MODEL}

The spectrum sensing can be viewed as a binary hypotheses test in which $\mathcal{H}_{0}$ denotes the hypothesis that the PU transmitter is off (inactive), whereas $\mathcal{H}_{1}$ denotes the hypothesis that the PU transmitter is on (active). The main metrics for assessing the performance of this test are the probability of false alarm, $P_{\text {fa, }}$, and the probability of detection, $P_{\mathrm{d}}$. The former is the probability of declaring an active PU transmitter when in fact it is inactive. The latter is the probability of declaring the PU transmitter active when it is indeed active. Mathematically,

$$
\begin{aligned}
P_{\text {fa }} & =\operatorname{Pr}\left\{\text { decision }=\mathcal{H}_{1} \mid \mathcal{H}_{0}\right\}, \\
P_{\mathrm{d}} & =\operatorname{Pr}\left\{\text { decision }=\mathcal{H}_{1} \mid \mathcal{H}_{1}\right\},
\end{aligned}
$$

where $\operatorname{Pr}\{A \mid B\}$ is the probability of the event $A$ conditioned on the occurrence of the event $B$.

When the SUs make local decisions on the presence of the PU signal to subsequently transmit them to the FC, a pair of probabilities of false alarm and detection will measure the local decision performance, and another pair will do the same with respect to the global decision performance made at the FC. For example, when the GLRT is used as the detection technique in each $\mathrm{SU}, P_{\text {faSU }}=\operatorname{Pr}\left\{T>\gamma \mid \mathcal{H}_{0}\right\}$ and $P_{\mathrm{dSU}}=\operatorname{Pr}\left\{T>\gamma \mid \mathcal{H}_{1}\right\}$ are the local metrics (for now assumed the same for all SUs), where $T$ is the test statistic and $\gamma$ is the local decision threshold. In this case, the global performance metrics are determined by the rule used to combine the local decisions at the FC. For example, let $u_{m}=0$ denote the decision made by the $m$-th SU associated to the hypothesis $\mathcal{H}_{0}, m=1,2, \ldots, M$, and $u_{m}=1$ associated to the hypothesis $\mathcal{H}_{1}$, with $M$ being the number of SUs in cooperation. For the combining rule $\mathrm{OR}$, the $\mathrm{FC}$ decides in favor of $\mathcal{H}_{1}$ if $\sum_{m=1}^{M} u_{m}>0$, and in favor of $\mathcal{H}_{0}$ otherwise; for the combining rule AND, the FC decides in favor of $\mathcal{H}_{1}$ if $\sum_{m=1}^{M} u_{m}=M$, and in favor of $\mathcal{H}_{0}$ otherwise; for the MAJ rule, the FC decides in favor of $\mathcal{H}_{1}$ if $\sum_{m=1}^{M} u_{m}>M / 2$, and in favor of $\mathcal{H}_{0}$ otherwise.

Under error-free report channels and equal local performances, the global metrics are determined from the local ones by means of [30]

$$
\begin{gathered}
P_{\mathrm{d}}=\sum_{l=k}^{M}\left(\begin{array}{c}
M \\
l
\end{array}\right)\left(P_{\mathrm{dSU}}\right)^{l}\left(1-P_{\mathrm{dSU}}\right)^{M-l}, \\
P_{\mathrm{fa}}=\sum_{l=k}^{M}\left(\begin{array}{c}
M \\
l
\end{array}\right)\left(P_{\mathrm{faSU}}\right)^{l}\left(1-P_{\mathrm{faSU}}\right)^{M-l} .
\end{gathered}
$$

The above expressions apply to the well-known $k$-out-of$M$ combining rule [30], in which the FC decides in favor of the presence of the PU signal if there are $k$ or more SUs that individually decide on the presence of the PU signal. When $k=1, k=M$, and $k=\lceil M / 2\rceil$, the $k$-out-of- $M$ rule specializes to the OR rule, the AND rule and the MAJ rule, respectively, where $\lceil M / 2\rceil$ is the smallest integer greater than or equal to $M / 2$. The general relations between the local and the global metrics when the local ones may be different from each other, and when the FC performs a weighted combining of the decisions of the SUs, can be obtained from [31, Eqns. (15) and (16)].

When the test statistic $T$ if formed at the FC, which is the case of both the GLRT and the CF-CPSC with data fusion, $P_{\text {fa }}=\operatorname{Pr}\left\{T>\gamma \mid \mathcal{H}_{0}\right\}$ and $P_{\mathrm{d}}=\operatorname{Pr}\left\{T>\gamma \mid \mathcal{H}_{1}\right\}$ are the global metrics, where $\gamma$ is now the global decision threshold set in the FC. 
The performance of the spectrum sensing is typically assessed by means of a receiver operating characteristic (ROC) curve, which trades $P_{\mathrm{fa}}$ versus $P_{\mathrm{d}}$. Low values of $P_{\mathrm{fa}}$ are desired to increase the throughput of the secondary network, while high values of $P_{\mathrm{d}}$ are desired to protect the primary network against interferences caused by the secondary terminals due to missed detections of the PU signal.

In order to improve the spacial diversity effect beyond the one provided by cooperation, and to reduce the sensing interval, assume that the $m$-th SU, $m=1,2, \ldots, M$, is equipped with $J$ antennas [32]. From each antenna, $N$ received samples are collected during a sensing interval and arranged in a matrix $\mathbf{Y}_{m} \in \mathbb{C}^{J \times N}$ given by

$$
\mathbf{Y}_{m}=\left[\begin{array}{llllll}
\mathbf{y}_{m, 1} & \mathbf{y}_{m, 2} & \cdots & \mathbf{y}_{m, j} & \cdots & \mathbf{y}_{m, J}
\end{array}\right]^{\mathrm{T}},
$$

where $\mathbf{y}_{m, j} \in \mathbb{C}^{N \times 1}$ is the vector formed with the $N$ samples collected by the $j$-th antenna, $j=1,2, \ldots, J$, of the $m$-th SU, and $[\cdot]^{\mathrm{T}}$ stands for matrix or vector transposition.

The discrete-time impulse response of the channel between the PU transmitter and the $j$-th antenna of the $m$-th SU can be represented by the vector $\mathbf{h}_{m, j} \in \mathbb{C}^{N_{\mathrm{c}} \times 1}$, where $N_{\mathrm{c}}$ is the impulse response length. The elements of $\mathbf{h}_{m, j}$ are the path gains, which are considered zero mean complex Gaussian random variables with unitary power gain, i.e. $\mathbb{E}\left[\mathbf{h}_{m, j}^{\dagger} \mathbf{h}_{m, j}\right]=1$, with $\mathbb{E}[\cdot]$ denoting the statistical expectation operator and $[\cdot]^{\dagger}$ denoting the conjugate and transpose operation. The path gains are kept constant during the sensing interval and are independently coined from one interval to the next. This channel model simulates a slow and time-varying frequencyselective Rayleigh fading.

It is assumed that when the PU signal is sensed, the channel output is already in a steady state, meaning that the received samples were affected by all path gains. In other words, the sensing interval does not encompass the tails of the channel output samples, which are transient periods at the beginning and the end of a PU transmission; such tails result from the influence of not all channel path gains and, thus, must be ignored. Then, the vectors $\mathbf{y}_{m, j}$ can be written as

$$
\mathbf{y}_{m, j}=\mathbf{R H}_{m, j} \mathbf{x}+\mathbf{v}_{m, j},
$$

where $\mathbf{H}_{m, j} \in \mathbb{C}^{\left(N+2 N_{\mathrm{c}}-2\right) \times\left(N+N_{\mathrm{c}}-1\right)}$ is a banded Toeplitz channel matrix whose band diagonal is formed from the elements of $\mathbf{h}_{m, j}$ [33], and $\mathbf{x} \in \mathbb{C}^{\left(N+N_{\mathrm{c}}-1\right) \times 1}$ represents the PU signal samples. The product $\mathbf{H}_{m, j} \mathbf{x}$ replaces the convolution between the transmitted signal and the channel impulse response. The additional product by the matrix $\mathbf{R}=\left[\begin{array}{lll}\mathbf{0}_{N \times\left(N_{\mathrm{c}}-1\right)} & \mathbf{I}_{N} & \mathbf{0}_{N \times\left(N_{\mathrm{c}}-1\right)}\end{array}\right]$ is responsible for cutting-off the above described tails in the channel output sequence, yielding the desired number $N$ of samples in $\mathbf{y}_{m, j}$. The block matrices forming $\mathbf{R}$ are two zero matrices of order $N \times\left(N_{\mathrm{c}}-1\right)$ and the identity matrix of order $N$. Finally, the vector $\mathbf{v}_{m, j} \in \mathbb{C}^{N \times 1}$ in (4) is formed by zero mean circularly-symmetric complex AWGN samples with variance $\sigma_{m}^{2}$. Notice that the noise variances are possibly different among the receivers of the SUs, which is a common situation in practice, but they are considered the same in each antenna branch, meaning that each SU antenna array is assumed to be calibrated by means of a suitable technique [34].
It is important to notice that the vector $\mathbf{x}$ in (4) could have any length greater than, or equal to $N+N_{\mathrm{c}}-1$, which would represent a PU transmission initiated more time before the beginning and after the end of the sensing interval. However, there is no reason for choosing such lengthened $\mathbf{x}$ in the present model, since only the $N$ steady-state samples are eventually considered.

For the sake of simplicity, no particular node distribution or mobility model is explicitly applied to the terminals of the cognitive secondary network. Nonetheless, node mobility is implicitly considered when dynamical received signal and noise powers are taken into account. In other words, when nonuniform noise and received signal powers are assumed, it can be interpreted that, due to node mobility, the SUs are at different distances from the FC in each sensing round. Moreover, if it is assumed that when at different locations the SUs are almost-surely subjected to different temperatures, the time-varying noise power also mimics the node mobility, as well as the inherent receiver component differences.

\section{Test Statistics And Fusion TeChniQues}

The variants of the GLRT and the CF-CPSC with respect to the way in which the spectrum sensing information is processed and transmitted to the FC are discussed in this section, assuming an error-free report channel.

\section{A. GLRT with sample fusion (SF) and weighted sample fusion (WSF)}

In the GLRT with SF, which can be referred to as the conventional cooperative GLRT [17], the $m$-th SU transmits the samples in $\mathbf{Y}_{m}$ to the FC; see (3). A new matrix $\mathbf{Y}_{\mathrm{FC}} \in \mathbb{C}^{J M \times N}$ is formed at the FC by concatenating the $M$ matrices $\left\{\mathbf{Y}_{m}\right\}$, according to

$$
\mathbf{Y}_{\mathrm{FC}}=\left[\begin{array}{llll}
\mathbf{Y}_{1}^{\mathrm{T}} & \mathbf{Y}_{2}^{\mathrm{T}} & \cdots & \mathbf{Y}_{M}^{\mathrm{T}}
\end{array}\right]^{\mathrm{T}} .
$$

The sample covariance matrix $\mathbf{R}_{\mathrm{FC}} \in \mathbb{C}^{J M \times J M}$ is then estimated as

$$
\mathbf{R}_{\mathrm{FC}}=\frac{1}{N} \mathbf{Y}_{\mathrm{FC}} \mathbf{Y}_{\mathrm{FC}}^{\dagger},
$$

from which the $J M$ ordered eigenvalues $\lambda_{1} \geq \lambda_{2} \geq \cdots \geq \lambda_{J M}$ are computed.

Since the FC does not know the noise variances $\left\{\sigma_{m}^{2}\right\}_{m=1}^{M}$, under the sample fusion approach it applies the GLRT test statistic [17]

$$
T_{\mathrm{SF}}=\frac{J M \lambda_{1}}{\sum_{i=1}^{J M} \lambda_{i}} .
$$

In order to improve the robustness of the GLRT with sample fusion against nonuniform noise, the authors of [1] and [23] developed two weighted sample fusion (WSF) schemes. In this paper, the scheme of [23] is used instead of the one in [1], since the former makes use of a maximum likelihood noise variance estimate, whereas the latter uses a less accurate estimate.

In the GLRT with weighted sample fusion, the matrix defined in (5) is modified to

$$
\mathbf{Y}_{\mathrm{FC}}^{\prime}=\left[\begin{array}{lll}
f_{1} \mathbf{Y}_{1}^{\mathrm{T}} f_{2} \mathbf{Y}_{2}^{\mathrm{T}} \cdots f_{M} \mathbf{Y}_{M}^{\mathrm{T}}
\end{array}\right]^{\mathrm{T}}
$$


where the weight $f_{m}$ is computed by the $m$-th SU according to $[23]$

$$
f_{m}=\frac{1}{\hat{\sigma}_{m}}=\left(\frac{1}{J-1} \sum_{j=2}^{J} \lambda_{j, m}\right)^{-\frac{1}{2}}
$$

with $\hat{\sigma}_{m}^{2}=\frac{1}{J-1} \sum_{j=2}^{J} \lambda_{j, m}$ being the maximum likelihood estimate of the noise variance at the input of the $m$-th $\mathrm{SU}$, assuming the presence of a signal [17], [35]. This estimate is made from the smallest $J-1$ ordered eigenvalues $\lambda_{2, m} \geq$ $\lambda_{3, m} \geq \cdots \geq \lambda_{J, m}$ of the $m$-th sample covariance matrix $\mathbf{R}_{m} \in \mathbb{C}^{J \times J}$, which is computed as

$$
\mathbf{R}_{m}=\frac{1}{N} \mathbf{Y}_{m} \mathbf{Y}_{m}^{\dagger}
$$

where $\mathbf{Y}_{m}$ comes from (3). The role of the weights $\left\{f_{m}\right\}$ is to weaken the signal samples that are subjected to higher noise levels and to strengthen those under lower noise levels, thus favoring a larger influence of the signal samples less affected by noise on the composition of the test statistic.

The modified sample covariance matrix $\mathbf{R}_{\mathrm{FC}}^{\prime} \in \mathbb{C}^{J M \times J M}$ is estimated at the FC as

$$
\mathbf{R}_{\mathrm{FC}}^{\prime}=\frac{1}{N} \mathbf{Y}_{\mathrm{FC}}^{\prime} \mathbf{Y}_{\mathrm{FC}}^{\prime \dagger}
$$

from which the $J M$ ordered eigenvalues $\lambda_{1}^{\prime} \geq \lambda_{2}^{\prime} \geq \cdots \geq \lambda_{J M}^{\prime}$ are computed. Thus, the GLRT test statistic given in [17], which is Eq. (7) of this paper, under the weighted sample fusion strategy becomes

$$
T_{\mathrm{WSF}}=\frac{J M \lambda_{1}^{\prime}}{\sum_{i=1}^{J M} \lambda_{i}^{\prime}}
$$

\section{B. GLRT with eigenvalue fusion $(E F)$ and weighted eigenvalue fusion (WEF)}

In this data fusion approach, the $m$-th SU terminal computes the sample covariance matrix (10) and its eigenvalues $\lambda_{1, m} \geq \lambda_{2, m} \geq \cdots \geq \lambda_{J, m}$. The $J M$ eigenvalues from all SUs are transmitted to the FC, where it is computed the test statistic of the modified GLRT with eigenvalue fusion, proposed originally in [36, Eq. (24)] and adapted here to the single-carrier PU transmission ${ }^{1}$, yielding

$$
T_{\mathrm{EF}}=\frac{J \sum_{m=1}^{M} \lambda_{1, m}}{\sum_{m=1}^{M} \sum_{j=1}^{J} \lambda_{j, m}} .
$$

Similarly to the weighted sample fusion, the weighted eigenvalue fusion proposed in [23] is also studied in this paper. In this case, more weight is given to eigenvalues coming from SUs affected by lower estimated noise powers, improving

\footnotetext{
${ }^{1}$ In [36] it is proposed an empirically-modified version of the eigenvalue-based GLRT given in [17], yielding the test statistic $T_{\mathrm{EF}, s}=\left(P J \sum_{m=1}^{M} \lambda_{1, s, m}\right)\left(\sum_{p=1}^{P} \sum_{m=1}^{M} \sum_{j=1}^{J} \lambda_{j, s, m}\right)^{-1}$ for centralized cooperative spectrum sensing of orthogonal frequency division multiple access (OFDMA) signals. The FC receives the $J P$ eigenvalues sent by the $M$ SUs and computes one decision for each sub-channel of the OFDMA signal, where $P$ is the total number of OFDMA sub-channels, $J$ is the number of eigenvalues of the sample covariance matrix, which is also the number of sub-carriers per sub-channel, denoted as $K^{\prime}$ in [36], and $s$ is the $s$-th OFDMA sub-channel.
}

the spectrum sensing robustness against nonuniform noise variances. The weights are given by

$$
g_{m}=f_{m}^{2}=\frac{1}{\hat{\sigma}_{m}^{2}},
$$

where $f_{m}$ is from (9). The resulting modified GLRT test statistic is $T_{\mathrm{WEF}}=\left(J \sum_{m=1}^{M} g_{m} \lambda_{1, m}\right)\left(\sum_{m=1}^{M} g_{m} \sum_{j=1}^{J} \lambda_{j, m}\right)^{-1}$, which after some simple manipulations [23] becomes

$$
T_{\mathrm{WEF}}=J\left(1+\frac{M}{\sum_{m=1}^{M} \frac{\lambda_{1, m}}{\sum_{j=2}^{J} \lambda_{j, m}}}\right)^{-1} .
$$

\section{GLRT with decision fusion (DF)}

When the GLRT with DF is considered, each SU makes its local decision upon the presence or absence of the PU signal and sends the decision to the FC. The GLRT test statistic [17] formed in the $m$-th SU is

$$
T_{\mathrm{DF}_{m}}=\frac{J \lambda_{1, m}}{\sum_{j=1}^{J} \lambda_{j, m}},
$$

where $\lambda_{1, m} \geq \lambda_{2, m} \geq \cdots \geq \lambda_{J, m}$ are the ordered eigenvalues of the $m$-th received signal sample covariance matrix given by (10). The $m$-th SU decision is $u_{m}=1$ if $T_{\mathrm{DF}_{m}}>\gamma$, and $u_{m}=0$ if $T_{\mathrm{DF}_{m}} \leq \gamma$, being $\gamma$ the local decision threshold. The global decision at the FC is then made according to the combining rule applied to the $M$ received decisions from the SUs, namely OR, AND or MAJ, as described in Section II.

\section{CF-CPSC with total sample fusion (TSF) and partial sample fusion (PSF)}

As already mentioned, the CF-CPSC [27] has low complexity and is robust against dynamical noise variance, likewise the original CPSC [25], but attains superior performance with respect to the original version. A single adaptation of the $\mathrm{CF}$ CPSC to work with SUs having multiple antennas is given here, since the CF-CPSC has been originally conceived to work with a single antenna per SU. The adaptation, which acts in the steps 1 to 4 in the subsequent algorithm, treats each antenna as a separate receiver, i.e., the CF-CPSC algorithm is applied to the signal received by each antenna branch, for each $\mathrm{SU}$, which from the algorithmic point of view is equivalent to having $M J$ single-antenna SUs instead of $M$ SUs with $J \geq 1$ antennas each. The adapted CF-CPSC method works according to the following steps:

1) Estimate the instantaneous power spectral density (PSD) of the discrete-time signal received at the $j$-th antenna of the $m$-th SU as

$$
\mathbf{F}_{m, j}^{\prime}=\frac{1}{N}\left|\operatorname{DFT}\left\{\mathbf{y}_{m, j}\right\}\right|^{2},
$$

where DFT $\{\cdot\}$ is the discrete Fourier transform (DFT) operator and $\mathbf{y}_{m, j}$ is from (4).

2) Compute the modified circular-even component of $\mathbf{F}_{m, j}^{\prime}$ according to

$$
\mathbf{F}_{m, j}[k]= \begin{cases}\frac{\mathbf{F}_{m, j}^{\prime}[1]+\mathbf{F}_{m, j}^{\prime}[N / 2+1]}{2}, & k=1, \\ \frac{\mathbf{F}_{m, j}^{\prime}[k]+\mathbf{F}_{m, j}^{\prime}[N-k+2]}{2}, & k=2,3, \ldots, N .\end{cases}
$$


3) Divide the sensed band into $L$ sub-bands and compute the signal power in the $\ell$-th sub-band, $\ell=1,2, \ldots, L$, as

$$
F_{\ell_{m, j}}=\sum_{k=1}^{V} \mathbf{F}_{m, j}[(\ell-1) V+k]
$$

where $V=N /(2 L)$.

4) Compute the total signal power in the sensed band,

$$
F_{\text {full }_{m, j}}=\sum_{k=1}^{N / 2} \mathbf{F}_{m, j}[k] .
$$

5) Compute the average of the ratio $F_{\ell_{m, j}} / F_{\text {full }_{m, j}}$, where the noise variance influence is canceled-out, yielding

$$
r_{m, \ell}=\frac{1}{J} \sum_{j=1}^{J} F_{\ell_{m, j}} / F_{\text {full }_{m, j}} .
$$

We emphasize that (21) is a more general computation in comparison with the corresponding one defined in [27], since here it is assumed that each SU has $J \geq 1$ antennas $\left(r_{m, \ell}=F_{\ell_{m}} / F_{\text {full }_{m}}\right.$ in [27], since $J=1$ for each SU).

6) For both the partial and the total sample fusion strategies, the adapted CF-CPSC test statistic for the $\ell$-th subband is formed at the FC, yielding

$$
T_{\mathrm{TSF} / \mathrm{PSF}}{ }_{\ell}=\frac{1}{M} \sum_{m=1}^{M} r_{m, \ell} .
$$

In TSF, the digitized samples from all vectors $\mathbf{y}_{m, j}$ are transmitted to the FC (see step 1), where all computations regarding the CF-CPSC method are carried-out. In PSF, the variables $r_{m, \ell}$ are computed by the SUs, then digitized and transmitted to the FC, thus transferring to the SUs most of the computations. On the other hand, less report channel data traffic is needed in PSF when compared to TSF.

7) Compare the test statistics with a global decision threshold $\gamma$ to reach the decision on the occupation state of the $\ell$-th sub-band, that is,

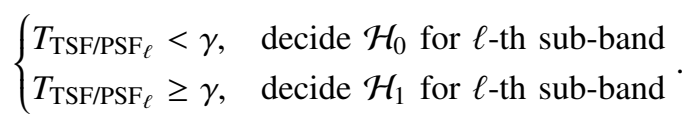

8) Finally, make the global decision on the occupation of the sensed band according to

$$
\left\{\begin{array}{ll}
\text { All sub-bands decided } \mathcal{H}_{0}, & \mathcal{H}_{0} \\
\text { One or more sub-bands decided } \mathcal{H}_{1}, & \mathcal{H}_{1}
\end{array} .\right.
$$

\section{Performance Results and Discussions}

In this section we present several MATLAB ${ }^{\circledR}$ simulation results regarding the global performance of the spectrum sensing in terms of ROCs. The aim of these results is to show the influence of having SUs under uniform and dynamicalnonuniform noise variances, as well as under different SNRs due to different distances from the PU transmitter, and different system parameters. Different sets of system parameters are explored, not necessarily but intensionally keeping some level of similarity with the parameters adopted in [1], since this paper is as an extension of it. The authors attest, however, that all the analyses discussed here could be made for any different and uncorrelated set of the system parameters considered here or in [1]. The GLRT and the CF-CPSC are analyzed under all fusion rules described in Section III.

In the case of uniform noise condition, the noise power for all SUs was $\sigma_{m}^{2}=1$, and the PU signal power was set according to the desired average SNR in each SU. In the nonuniform-dynamical noise condition, the noise power was varied uniformly within $\pm 80 \%$ around the average $\bar{\sigma}_{m}^{2}=1$, that is, $\sigma_{m}^{2} \sim \mathcal{U}[0.2,1.8]$. The variations occur among SUs and from one sensing interval to the next.

It is important to highlight that the uniform distribution for the noise variance fluctuations in each SU was chosen here for simplicity; other distributions could be adopted as well, for instance some distribution based on field measurements or on device-dependent models.

The average signal power received by the $m$-th SU was computed according to

$$
P_{m}=\bar{\sigma}_{m}^{2} \times 10^{\mathrm{SNR}_{m} / 10}=10^{\mathrm{SNR}_{m} / 10},
$$

with the average SNR in each SU configured according to Table I. In this table, the SNRs are shown for $M=3$ SUs equipped with $J=6$ antennas each, and for $M=6$ SUs with $J=3$ antennas. Notice that the average of the SNR values is $-10 \mathrm{~dB}$, which represents a challenging situation from the perspective of the spectrum sensing. For instance, the IEEE 802.22 WRAN standard [28], [29] determines that the sensors have to detect very weak primary signals, for instance with power level of $-114 \mathrm{dBm}$, with a probability of detection of 0.9, which represents a low SNR situation for this target performance.

TABLE I: Average SNRs for the SUs, assuming $M=3$ SUs having $J=6$ antennas each, and $M=6$ SUs having $J=3$ antennas each.

\begin{tabular}{lcccccc}
\hline \hline \multicolumn{7}{c}{ SNR for the $m$-th SU, dB } \\
\hline \hline & $m=1$ & $m=2$ & $m=3$ & $m=4$ & $m=5$ & $m=6$ \\
\hline \hline$M=3$ & -12 & -10 & -8 & & & \\
$M=6$ & -15 & -13 & -11 & -9 & -7 & -5 \\
\hline \hline
\end{tabular}

The PU transmitted signal vector $\mathbf{x}$ was configured to simulate base-band binary phase-shift keying (BPSK) symbols [37], each one represented by 5 samples. The channel between the PU transmitter and the receivers of the SUs was set to simulate a slow, multi-path frequency-selective Rayleigh fading according to the delay profile given in Table II, which follows the IEEE 802.22 standard for WRAN scenarios [28], [29]. In other words, the vectors $\mathbf{h}_{m, j}$ that model the channels between the PU transmitter and the $j$-th antenna of the $m$-th SU are composed of elements following a complex Gaussian distribution whose power gain is determined from Table II. The overall power gain was then normalized to unit.

TABLE II: Delay profile of the PU-SU multi-path fading channel.

\begin{tabular}{lrrrrrr}
\hline \hline Path index & 1 & 2 & 3 & 4 & 5 & 6 \\
\hline \hline Path delay, $\mu$ s & 0 & 3 & 8 & 11 & 13 & 21 \\
Path gain, dB & 0 & -7 & -15 & -22 & -24 & -19 \\
\hline \hline
\end{tabular}


Each pair of global $P_{\mathrm{fa}}$ and $P_{\mathrm{d}}$ in the ROC curves presented hereafter was generated from 100,000 Monte Carlo runs comprising the events: primary signal transmission, spectrum sensing, fusion and global decision. The PU signal activity was simulated by means of a Bernoulli random variable with probability of success equal to 0.5 , yielding a $50 \%$ PU signal duty-cycle.

In the case of the CF-CPSC, the number of sub-bands dividing the sensed band was set to $L=5$.

The report channel was assumed perfect, meaning that no transmission errors were caused to the information sent by the SUs to the FC in any of the fusion strategies.

Figure 1 shows performance results for the GLRT and the CF-CPSC under nonuniform non-dynamical noise condition, with noise variances randomly selected but constant over all sensing intervals. This non-dynamical noise variance approach was adopted in [1], but can lead to non-general conclusions regarding absolute or relative performances, because the curve positions may change significantly from a given realization of the set of noise variances to another, even if the average variance is maintained. This is demonstrated by the noticeable difference between the results in Fig. 1(a) with respect to those in Fig. 1(b). These results were obtained assuming $M=3$ SUs with $J=6$ antennas each, $N=30$ received signal samples per antenna in each sensing interval, yielding $J N=180$ samples per SU, noise variances $\left(\sigma_{1}^{2}, \sigma_{2}^{2}, \sigma_{3}^{2}\right)=(1.75,0.3,0.95)$ in the case of Fig. 1(a) and $\left(\sigma_{1}^{2}, \sigma_{2}^{2}, \sigma_{3}^{2}\right)=(1.1,1.2,0.7)$ in the case of Fig. 1(b). Notice that the average noise variance among the SUs is equal to 1 . The average variance for the $m$-th SU in several random realizations of such sets of variances over time would be $\bar{\sigma}_{m}^{2}=1$, yielding those SNRs in Table I, i.e., -12 , -10 and $-8 \mathrm{~dB}$. However, the actual SNRs achieved by fixing the above noise variances over time are $-14.43,-4.77$ and $-7.78 \mathrm{~dB}(-8.99 \mathrm{~dB}$ on average) for Fig. 1(a) and -12.41 , -10.79 and $-6.45 \mathrm{~dB}(-9.88 \mathrm{~dB}$ on average) for Fig. 1(b).

From the results in Fig. 1 one can notice that not only the performances of all techniques have changed from Fig. 1(a) to Fig. 1(b), but also the ranking has changed. This can be observed, for instance, in the case of the GLRT with DF and combining rule MAJ, whose performance is better than the GLRT with EF and the GLRT with SF in Fig. 1(a), but is worse than these two in Fig. 1(b). As another example, in Fig. 1(a) the GLRT with SF is the worst, but in Fig. 1(b) it is only outperformed by the GLRT with WSF and the CF-CPSC. The same behavior can be observed in the case of the GLRT with DF and combining rule AND, for which the performance overcomes the GLRT with SF in Fig. 1(a), but is far below it in Fig. 1(b).

The results in Fig. 1 bring out the important conclusion that it is not encouraged to assess the spectrum sensing performance under nonuniform noise conditions by keeping the noise variances unchanged from one sensing interval to the next, unless this behavior is intended to mimic some particular scenario that will actually happen in practice or is worth analyzing for some specific purpose. Nonetheless, this fixed noise variance condition is still useful for determining to which extent a given technique is robust against nonuniform noise. However, not a single result should be used. For instance, a record of the average performances and their variations over several realizations of the noise powers is capable of reveal the specific degree of robustness of the techniques.

Table III shows averages and standard deviations of the area under the ROC curve (AUC) for each technique under analysis, and the performance ranking. The top-three are emphasized in bold. The AUCs were obtained from 5000 uniformly-distributed realizations of the noise variances in the interval $[0.2,1.8]$, under the same system parameters used to plot Fig. 1. Each realization of the noise variances was kept unchanged over 1000 sensing intervals. The performance ranking shows that the top-three techniques are the GLRT with WSF, the CF-CPSF with TSF and PSF, and the GLRT with WEF (which has attained roughly the same performance of the GLRT with DF OR). The poorest performance was achieved by the GLRT with DF AND. On the other hand, it can be seen that the most robust technique is the CF-CPSC, for which the smallest standard deviation of the AUCs has been achieved. The CF-CPSC is closely followed by the GLRT with WSF and the GLRT with DF AND. The less robust technique is the GLRT with DF OR. From Table III it can also be concluded that none of the techniques under analysis wins over the others in terms of both the performance and the robustness against nonuniform noise. However, one can clearly notice that the CF-CPSC and the GLRT with WSF are equivalently attractive in terms of these two metrics.

TABLE III: Averages and standard deviations of AUCs over several realizations of the noise variances. System parameters are those used to plot Fig. 1.

\begin{tabular}{lcc}
\hline \hline & Average AUC; ranking & Std. deviation; ranking \\
\hline GLRT, SF & $0.8028 ; 6$ & $0.0950 ; 6$ \\
GLRT, WSF & $0.9301 ; \mathbf{1}$ & $0.0659 ; \mathbf{2}$ \\
GLRT, EF & $0.8209 ; 5$ & $0.0812 ; 4$ \\
GLRT, WEF & $0.8650 ; \mathbf{3}$ & $0.0977 ; 7$ \\
GLRT, DF OR & $0.8574 ; 4$ & $0.1024 ; 8$ \\
GLRT, DF MAJ & $0.7871 ; 7$ & $0.0875 ; 5$ \\
GLRT, DF AND & $0.6997 ; 8$ & $0.0663 ; \mathbf{3}$ \\
CF-CPSC, TSF/PSF & $0.9071 ; \mathbf{2}$ & $0.0643 ; \mathbf{1}$ \\
\hline \hline
\end{tabular}

In practice, the SNR may vary due to the mobility of the SUs (which causes variations in the average received signal power), and due to noise power variations caused by different temperatures to which the SUs are submitted at different locations and in different moments. Thus, to avoid misinterpretations in the spectrum sensing performance analyses and to confer a more practical appeal to them, hereafter it is considered that the noise powers vary from one sensing interval to the next. We refer to this as a nonuniformdynamical noise situation.

Figure 2 shows the performances of the GLRT and the CF-CPSC under uniform, and nonuniform-dynamical noise conditions, assuming different SNRs among the SUs, as defined in Table I. Different numbers of SUs and antennas are also considered, with a fixed number of samples collected by each SU, i.e. $J N=180$ samples. The number of SUs, $M$, and the number of antennas, $J$, per SU were chosen in such a way that the product $J M=18$ is fixed for both scenarios. With this constraint it is possible to establish a fair performance comparison from the point of view of the influence of the number of antennas in each SU and the 

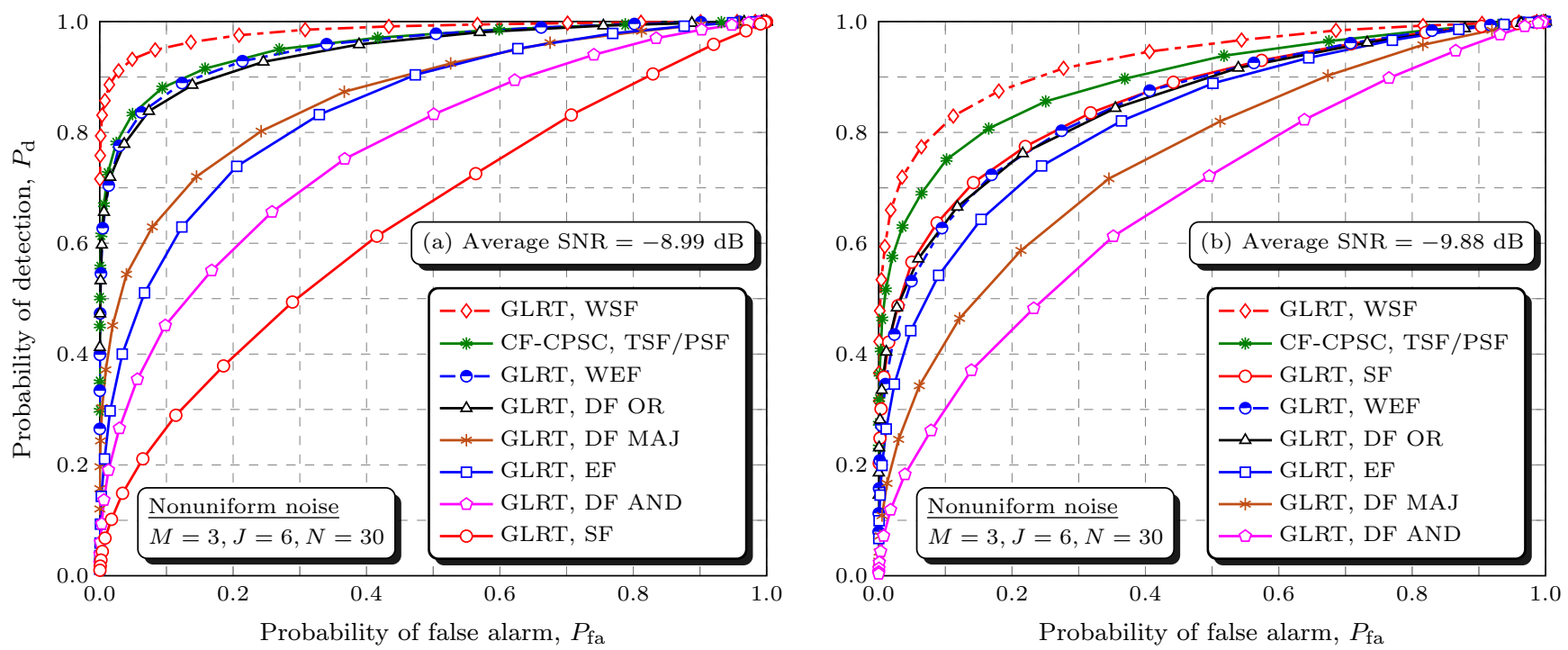

Fig. 1: Global spectrum sensing performance under nonuniform noise for the CF-CPSC with total sample fusion and partial sample fusion (TSF/PSF), and for the GLRT with sample fusion (SF), weighted sample fusion (WSF), eigenvalue fusion (EF), weighted eigenvalue fusion (WEF), and decision fusion (DF) under the combining rules OR, AND and majority-voting (MAJ), assuming $M=3$ SUs with $J=6$ antennas each and $N=30$ samples per antenna. The noise variances were kept unchanged over all sensing intervals, as in [1], a set to $\left(\sigma_{1}^{2}, \sigma_{2}^{2}, \sigma_{3}^{2}\right)=(1.75,0.3,0.95)$ in (a) and $\left(\sigma_{1}^{2}, \sigma_{2}^{2}, \sigma_{3}^{2}\right)=(1.1,1.2,0.7)$ in (b).

number of cooperating SUs in the secondary network. In other words, it is possible to infer if it is better, in terms of performance, having more SUs with less antennas each or less SUs with more antennas each. One can notice, however, that the same analysis could be performed for other numbers of SUs and antennas per SU, provided that the product $J M$ is maintained. Furthermore, the value of $J M=18$ was adopted in this paper to maintain coherence with the configurations implemented in the conference paper [1], which is the work on which the present research was based. Such a number represents a desired practical scenario in which the number of SUs in cooperation and the number of antennas in each SU are not high.

Figures 2(a) and (b) were built by adopting $M=3$ SUs, each containing $J=6$ antennas, and $N=30$ samples collected in each antenna branch, for the uniform and the nonuniformdynamical noise scenarios, respectively. On the other hand, Figs. 2(c) and (d) were built by adopting $M=6$ SUs, each containing $J=3$ antennas, and $N=60$ samples for each antenna, also for the uniform and for the nonuniformdynamical noise scenarios, respectively. In the uniform noise scenario, i.e., Figs. 2(a) and (c), $\sigma_{m}^{2}=1$ for all SUs. In the nonuniform-dynamical noise scenario, i.e., Figs. 2(b) and (d), $\sigma_{m}^{2} \sim \mathcal{U}_{m}[0.2,1.8]$ for any $m$. Notice that, contrasting to what has been observed regarding the results shown in Fig. 1, the average SNR given in Table I for each SU is attained.

From graphs (a) and (b) of Fig. 2, one can see that only the GLRT with SF, i.e, the conventional GLRT, has been penalized by the nonuniform-dynamical noise situation. The other techniques benefited from this situation, but in different degrees. Recalling that noise variances are uniformly distributed around 1 among the SUs and over time, the observed improvements can be credited to lower penalties caused by variances above 1 than ameliorations caused by variances below 1. In other words, based on the observation of the simulation results presented in the graphs (a) and (b) of Fig. 2, a given noise variance fluctuation above the mean reduces the performance less than what the same fluctuation below the mean improves it. An inverse behavior applies to the SNR when it is not uniform among the SUs.

A similar behavior can be observed from the graphs (c) and (d) of Fig. 2, but now with smaller improvements in the nonuniform-dynamical noise condition, with the exception of the GLRT with SF, which again has been strongly penalized. Moreover, a small performance reduction for the GLRT with $\mathrm{EF}$ in the nonuniform noise condition is observed, which is credited to the smaller number of antennas (yielding a smaller covariance matrix order) in comparison with the cases considered in graphs (a) and (b).

It can also be noticed from all graphs in Fig. 2 that the CF-CPSC is intrinsically robust against nonuniform-dynamical noise, as expected. It can also be concluded that the sample and eigenvalue weighting schemes (WSF and WEF, respectively) have brought a remarkable robustness improvement to the GLRT. The larger the noise variance differences, the more effective is the weighting process.

An interesting aspect unveiled by Fig. 2 is that more SUs with less antennas can yield better performances than less SUs with more antennas. This would be even more emphasized if the SUs were subjected to correlated shadowing, a situation in which the close apart antenna array elements are less efficient in terms of spatial diversity than far apart SUs.

The results in Figs. 1 and 2 show that the GLRT with DF and combining rules OR, MAJ and AND achieve quite different performances, which is credited to the higher probabilities of correct local decisions made by the SUs under high SNRs, and the higher probabilities of incorrect local decisions made by the SUs under low SNRs. For instance, notice that the OR rule is superior, since a single SU yielding correct decisions more frequently (under high SNR) is enough for improving 

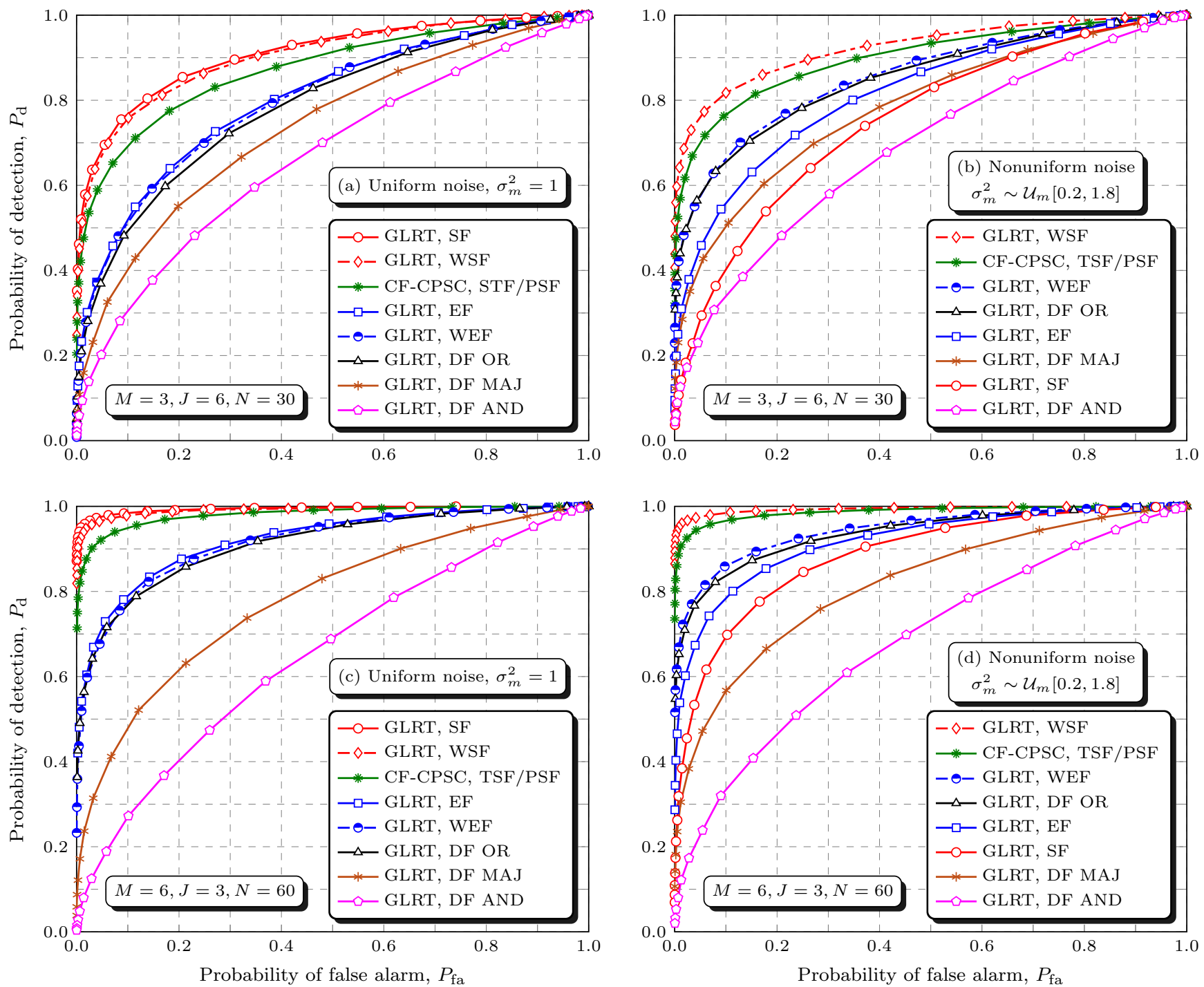

Fig. 2: Global spectrum sensing performance under uniform noise and nonuniform-dynamical noise for the CF-CPSC with total sample fusion and partial sample fusion (TSF/PSF), and for the GLRT with sample fusion (SF), weighted sample fusion (WSF), eigenvalue fusion (EF), weighted eigenvalue fusion (WEF), and decision fusion (DF) under the combining rules OR, AND and majority-voting (MAJ). Graphs (a) and (b) are for $M=3, J=6, N=30$. Graphs (c) and (d) are for $M=6, J=3, N=60$. In the nonuniform-dynamical noise scenario, noise variances are uniformly distributed among SUs and independently varied from one sensing interval to the next.

the global decision accuracy, because a single correct decision determines the correctness of the global decision. On the other hand, a single SU yielding incorrect decisions more frequently (low SNR) is enough for reducing the global decision accuracy in the case of the AND rule. The MAJ is in-between these two extreme cases, since a correct global decision is reached based on the majority of the correct decisions made by the SUs.

From the pair of graphs $(a, b)$ to the pair $(c, d)$ of Fig. 2 it can be noticed that all techniques, except the GLRT with DF AND, have improved their performances. The reason for the unaltered performance of the GLRT with DF AND can be explained in light of the previous paragraph. The improvement achieved by the other techniques can be justified by the following arguments: i) the product $J M$ is fixed, which means that the diversity effect is the same in both cases; ii) the reduction in the order $J \times J$ of the sample covariance matrix from $(\mathrm{a}, \mathrm{b})$ to $(\mathrm{c}, \mathrm{d})$ is somewhat compensated for by the increased number $N$ of samples used to estimate this matrix from $(a, b)$ to $(c, d)$ in the DF, EF and WEF schemes; iii) the remaining schemes make use of a fixed number $N J$ of samples, which put them into the same situation from $(a, b)$ to $(\mathrm{c}, \mathrm{d})$; iv) thus, the improvements are credited to the alreadymentioned unbalanced influence of the nonuniform SNRs, i.e., an SNR shift above the mean improves the performance less than what the same shift below the mean reduces it (notice from Table I that the SNR shifts above and below the mean $-10 \mathrm{~dB}$ are the same for the two cases analyzed).

Table IV lists the techniques and their relative positions ranked according to the ROC metric extracted from Fig. 2, that is, the performance of a given technique is superior with respect to another one if, for a given probability of false alarm, it attains the highest probability of detection. The bold-faced numbers highlight the top-three techniques in each condition. It can be seen that the top-three are the same for $M=3$ and $J=6$ and for $M=6$ and $J=3$. The ranking has changed from the uniform noise to the nonuniform-dynamical 
noise condition, as well as the performance shifts. The poorest performance in all situations was attained by the GLRT with DF and combining rule AND, and the largest performance shift was demonstrated by the GLRT with SF. The smallest shift was achieved by the GLRT with WSF, closely followed by the GLRT with EF and the CF-CPSC. Notice that the topthree performances shown in the third column of Table IV are the same as those unveiled by the average AUCs in Table III, which was constructed under the same settings, but used the average AUC instead of the absolute ROC position for establishing the relative performance positions. Changes in some ranking positions in Table IV with respect to the performance ranking in Table III are credited to normal statistical fluctuations of the Monte Carlo simulation results.

TABLE IV: ROC performance ranking of the techniques under analysis in the uniform, and nonuniform-dynamical noise conditions. Bold-faced numbers highlight the top-three techniques in each condition.

\begin{tabular}{lcccc}
\hline \hline & \multicolumn{2}{c}{ Uniform noise } & \multicolumn{2}{c}{ Nonuniform noise } \\
\hline \hline & $M=3$ & $M=6$ & $M=3$ & $M=6$ \\
& $J=6$ & $J=3$ & $J=6$ & $J=3$ \\
\hline \hline GLRT, SF & $\mathbf{1}$ & $\mathbf{1}$ & 7 & 6 \\
GLRT, WSF & $\mathbf{2}$ & $\mathbf{2}$ & $\mathbf{1}$ & $\mathbf{1}$ \\
GLRT, EF & 4 & 4 & 5 & 5 \\
GLRT, WEF & 5 & 5 & $\mathbf{3}$ & $\mathbf{3}$ \\
GLRT, DF OR & 6 & 6 & 4 & 4 \\
GLRT, DF MAJ & 7 & 7 & 6 & 7 \\
GLRT, DF AND & 8 & 8 & 8 & 8 \\
CF-CPSC, TSF/PSF & $\mathbf{3}$ & $\mathbf{3}$ & $\mathbf{2}$ & $\mathbf{2}$ \\
\hline \hline
\end{tabular}

The ROC performance analysis was also undertaken for the case in which the channels between the PU transmitter and the receivers of the SUs are considered flat instead of frequencyselective. The corresponding ROC results are not presented for the sake of conciseness, and because they would not bring any relevant information besides the following ones: the performances of all techniques improve from the frequencyselective to the flat fading channel, as expected, maintaining the ranking. The CF-CPSC is less sensitive to the channel model, meaning that its performance changes slightly from the frequency-selective to the flat fading condition. The sensitivity of the CF-CPSC is lower when the number of SUs is $M=6$ than when $M=3$. If the SNRs among the receivers of the SUs are considered uniform, the performances of all techniques reduce slightly when $M=3, J=6$. A more noticeable reduction, though still small, occurs when $M=6, J=3$. The exception is the GLRT with DF and combining rule AND, for which the performance practically does not change from the uniform to the nonuniform SNR condition.

\section{TRADE-OFF AMONG PERFormance, REPORTING CHANNEL TRAFFIC AND COMPUTATIONAL COMPLEXITY}

As far as the volume of data traffic over the report channel is concerned, the GLRT with SF is equivalent to the GLRT with WSF, and the GLRT with EF is equivalent to the GLRT with WEF, since the weighting operation does not influence the traffic because it is implemented by the SUs in both cases, just producing modified samples and eigenvalues to be transmitted to the FC. We highlight that the weighting operation of the GLRT with WEF can be applied directly by the FC, what can be concluded from (15).
In the case of the GLRT with SF/WSF, the $M$ SUs, each having $J$ antennas, collect $N$ samples per antenna, yielding $M J N$ samples that are subsequently sent to the FC. Since in practice the real and imaginary parts of the sample values are treated by the two branches of a quadrature transceiver, each branch will apply a $b$-bit quantization process, yielding a total of $2 b M J N$ bits reported to the FC in each sensing round.

In GLRT with EF/WEF, each SU computes $J$ eigenvalues of the received signal sample covariance matrix of order $J \times J$, and transmits these eigenvalues or their weighted versions to the FC. Assuming the same number $b$ of quantization bits, the total data traffic reported to the FC is $b M J$ bits per sensing round. Notice that the EF/WEF yields $2 b M J N / b M J=2 N$ times less bits than the SF/WSF.

For the CF-CPSC with TSF, the test statistic is completely implemented at the FC from the samples sent by the SUs. Thus, the report channel traffic per sensing round is the same as in the case of the SF/WSF, that is, $2 b M J N$ bits.

In the case of the CF-CPSC with PSF, the values of $r_{m, \ell}$ in (22) are computed by the $m$-th SU for all sub-bands $\ell=$ $1,2, \ldots, L$. The $M L$ resulting values are transmitted to the FC, where the final test statistic is formed. Thus, the report channel traffic is $b M L$ bits per sensing round.

The ratio between the report channel traffics of the GLRT with SF/WSF and the CF-CPSC with PSF is $2 b M J N / b M L=$ $2 J N / L$, meaning that the latter saves more report channel resources if $L<2 J N$, which can be easily accomplished in practice. On the other hand, the ratio between the traffics of the GLRT with EF/WEF and the CF-CPSC with PSF is $b M J / b M L=J / L$, meaning that the latter saves more report channel resources if $L<J$, which is not impossible to achieve in practice (recall that in the simulation results we have considered $L=5$, and $J=6$ or $J=3$ ).

In what concerns the GLRT with all decision fusion schemes, namely the DF with OR, AND and MAJ rules, only $M$ bits are transmitted to the FC per sensing round, yielding the smallest report channel traffic. The GLRT with $\mathrm{SF} / \mathrm{WSF}$ and the GLRT with EF/WEF reach a traffic that is $2 b M J N / M=2 b J N$ and $b M J / M=b J$ times higher, respectively. The CF-CPSC with PSF achieves a traffic ratio of $b M L / M$ with respect to the DF strategies, occupying more report channel resources, since $b L>1$ by definition.

To facilitate comparisons, Table $\mathrm{V}$ summarizes the above total number of bits transmitted to the FC for each technique, as well as the associated traffic ratios.

TABLE V: Total number of reported bits and traffic ratios. (1) GLRT with SF/WSF and CF-CPSC with TSF; (2) GLRT with EF/WEF; (3) CF-CPSC with PSF; (4) GLRT with $\mathrm{DF}$. The row and column heads are the technique and the corresponding number of bits.

\begin{tabular}{lllll}
\hline \hline \multicolumn{5}{c}{ Traffic ratios (rows divided by columns) } \\
\hline \hline & (1), $2 b M J N$ & (2), $b M J$ & (3), $b M L$ & (4), $M$ \\
\hline \hline (1), $2 b M J N$ & 1 & $2 N$ & $2 J N / L$ & $2 b J N$ \\
(2),$b M J$ & $1 / 2 N$ & 1 & $J / L$ & $b J$ \\
(3),$b M L$ & $L / 2 J N$ & $L / J$ & 1 & $b L$ \\
(4), $M$ & $1 / 2 b J N$ & $1 / b J$ & $1 / b L$ & 1 \\
\hline \hline
\end{tabular}

A numerical example might be of further help at this point. Assume that the analog quantities to be reported are digitized with $b=4$ bits, and that the techniques (1) (GLRT 
with SF/WSF and CF-CPSC with TSF) apply quadrature phase-shift keying (QPSK) modulation [37] in the report transmissions, so that each symbol carries one bit related to the real part of a sample value, and the other related to the imaginary part. Consider that the techniques (2) (GLRT with EF/WEF), (3) (CF-CPSC with PSF) and (4) (GLRT with DF) apply BPSK modulation for the report channel transmissions. The two system configurations analyzed in Section IV were $M=3, J=6, N=30$, and $M=6, J=3, N=60$. The number of sub-bands in the CF-CPSC was $L=5$. Adopting these configurations, the number of symbols transmitted to the FC per sensing round and the associated traffic ratios determined according to Table $\mathrm{V}$ are shown in Tables VI and VII, respectively.

TABLE VI: Total number of modulation symbols and traffic ratios. (1) GLRT with SF/WSF and CF-CPSC with TSF; (2) GLRT with EF/WEF; (3) CF-CPSC with PSF (4) GLRT with DF. The row and column heads are the technique and the corresponding number of symbols, for $M=3, J=6$ and $N=30$.

\begin{tabular}{lllll}
\hline \hline \multicolumn{5}{c}{ Traffic ratios (rows divided by columns) } \\
\hline \hline & (1), 2160 & (2), 72 & (3), 60 & (4), 3 \\
\hline \hline (1), 2160 & 1 & 30 & 36 & 720 \\
(2), 72 & $1 / 30$ & 1 & $6 / 5$ & 24 \\
(3), 60 & $1 / 36$ & $5 / 6$ & 1 & 20 \\
(4), 3 & $1 / 720$ & $1 / 24$ & $1 / 20$ & 1 \\
\hline \hline
\end{tabular}

TABLE VII: Total number of modulation symbols and traffic ratios. (1) GLRT with SF/WSF and CF-CPSC with TSF; (2) GLRT with EF/WEF; (3) CF-CPSC with PSF; (4) GLRT with DF. The row and column heads are the technique and the corresponding number of symbols for $M=6, J=3$ and $N=60$.

\begin{tabular}{lllll}
\hline \hline \multicolumn{5}{c}{ Traffic ratios (rows divided by columns) } \\
\hline \hline & (1), 4320 & (2), 72 & (3), 120 & (4), 6 \\
\hline \hline (1), 4320 & 1 & 60 & 36 & 720 \\
(2), 72 & $1 / 60$ & 1 & $3 / 5$ & 12 \\
(3), 120 & $1 / 36$ & $5 / 3$ & 1 & 20 \\
(4), 6 & $1 / 720$ & $1 / 12$ & $1 / 20$ & 1 \\
\hline
\end{tabular}

From Tables VI and VII one can conclude that it is better to have more SUs (large $M$ ) with less antennas (small $J$ ) than less SUs with more antennas if the report channel traffic is analyzed solely, except in the case of the technique (2) (GLRT with EF/WEF), in which the number of eigenvalues sent to the FC does not change if the product $M J$ is maintained. Nonetheless, the diversity gain produced with multiple antennas might be far below the diversity gain produced by spatially separated SUs, especially in scenarios of spatially-correlated shadowing.

The high number of symbols transmitted when the sample fusion (1) is adopted highlights an important drawback of this scheme. The techniques (2) (GLRT with EF/WEF) and (3) (CFCPSC with PSF) are similar and considerably better in terms of the volume of report channel traffic when compared to (1), but still occupy significantly more report channel resources when compared with the decision fusion scheme adopted by the technique (4) (GLRT with DF).

Regarding the computational complexity, the techniques differ in terms of the amount of computations and the number of samples stored and processed. For the GLRT with SF and the CF-CPSC with TSF, most of the computational burden is located at the FC, since it has to process $2 b M J N$ bits. In the case of the GLRT with SF, the FC has to build the sample covariance matrix and estimate $J M=18$ eigenvalues to compute the test statistic (7), subsequently making the global decision. For the CF-CPSC with TSF, the algorithm processes the same number of samples to yield the test statistic (22) and the subsequent global decision. As highlighted in [25], the computational complexities for estimating the eigenvalues in the GLRT and for working with the Fourier transformations used by the CF-CPSC are comparable, with a small advantage of the CF-CPSC. It is worth noticing that, for these two techniques, the computational burden in the SUs is low, since they have only to digitize and transmit the sample values.

Considering the GLRT with WSF, the computational complexity at the FC is even higher than in the case of the GLRT with SF, since the calculation and application of the weights (9) are additional to the computations of the GLRT with SF, before the test statistic (12) is formed.

The computational complexity of the SUs increases with respect to the previous cases when the GLRT with EF or WEF is applied, since each SU must handle the $J N$ sample values to build its sample covariance matrix. On the other hand, the computational burden of the FC is reduced, since it has to handle only $b M J$ bits and compute the test statistic (13) or (15). Notice that no significant additional burden due to the computation of the weights is needed in the case of the GLRT with WEF with respect to the EF, since all eigenvalues are already available to the FC.

For the CF-CPSC with PSF, the computational burden is shifted from the FC to the SUs, where the partial statistics (21) are computed. The $\mathrm{FC}$ receives these statistics and just have to compute (22) and make the global decision.

The GLRT with DF is advantageous in terms of report channel traffic, but this is the technique with the highest computational complexity in the SUs, since each one has to compute the test statistic (16) for subsequent local decision and transmission to the FC. Then, one must trade the report traffic against the complexity. The former is more relevant in networks with limited report channel resources, which is aggravated when the number of SUs in cooperation increases. The latter is more relevant when lightweight and low power consumption of the SU terminals are desired.

The global spectrum sensing performance must also be included in the trade-off analysis. For example, the CF-CPSC with PSF achieves good performances and robustness against nonuniform noise powers and SNRs, demanding less report channel traffic with respect to the GLRT with SF/WSF and comparable traffic with respect to the GLRT with EF/WEF.

When high spectrum sensing performance as well as low complexity of the SUs are targeted, no matter the report channel traffic and the robustness under nonuniform noise and SNRs, the GLRT with WSF is an attractive solution. If the complexity of the SUs can be increased, the GLRT with WEF will bring robustness against nonuniform noise and SNRs.

On the other hand, the attractiveness of the GLRT with DF with respect to the low report channel traffic is somehow blurred by the poor performances of the DF under the MAJ and the AND combining rules.

The GLRT with SF is the less attractive solution in the nonuniform noise scenario due to its strong performance 
degradation. Yet, the report channel traffic is very high. Its single advantage is the good performance under uniform noise, and low computational complexity at the SUs.

It is worth recalling that the report channels were assumed error-free, meaning that they do not cause any lost to the data sent by the SUs to the FC. In practice, however, these channels, as any communication channel, are intrinsically erroneous, which degrades the global spectrum sensing performance. Imperfect report channels are expected to degrade $P_{\text {fa }}$ and $P_{\mathrm{d}}$ in different degrees depending on the adopted technique [38][41]. Based on these references, it can be concluded that the sensitivity to channel errors increases as the amount of data transmitted from the SUs to the FC in each reporting round reduces. For example, it is expected that decision fusion strategies are more prone to report channel errors than the fusion of digitized received signal samples or related quantities.

One might resort to the use of forward error correcting (FEC) codes for protecting the transmissions from the SUs to the FC, as done in [42]-[45], which increases the computational complexity at the SUs and at the FC due to the encoding and decoding processes, respectively. However, the amount of bits added to protect the transmissions must be traded against the overall volume of data that can be sent to the FC, since the reporting time interval is limited.

If exactly the same FEC scheme is applied to all the techniques analyzed in the present paper, no matter the specific code, the ratios in Table $\mathrm{V}$ are maintained, as well those in Table VI and in Table VII. This means that the ranking is maintained in terms of report channel traffic, being the DF the most advantageous and the GLRT with SF/WSF and CFCPSC with TSF the most disadvantageous. For the GLRT with $\mathrm{EF} / \mathrm{WEF}$ and the CF-CPSC with PSF, the final ranking will depend on the number of sub-bands, $L$, of the CF-CPSC.

\section{CONCLUSIONS AND OPPORTUNITIES FOR FURTHER RESEARCH}

This paper presented an analysis of the performance, the report channel traffic and the computational complexity of two blind centralized cooperative spectrum sensing schemes: the eigenvalue-based GLRT and the CF-CPSC, under the effect of unequal and dynamical received signal and noise powers. These schemes were the basis for constructing the following variations: GLRT with sample fusion and weighted sample fusion, GLRT with eigenvalue fusion and weighted eigenvalue fusion, GLRT with decision fusion under the combining rules OR, AND and MAJ, CF-CPSC with total sample fusion and CF-CPSC with partial sample fusion. Amongst several particular conclusions, it has been shown that there is no technique superior to the others in terms of all metrics. As a consequence, the choice of the most suitable technique must be carefully made to match the specific scenario to which the technique is aimed to be applied. The results presented in this paper is intended to serve as a guideline for assisting this choice.

Some opportunities for future related research are: i) to include errors in the report channel (we conjecture that some techniques might be more vulnerable to these errors, especially those that achieve less report channel traffic, i.e., less bits carry the important spectrum sensing information); ii) to consider that the primary signal starts and finishes its transmission at any time, meaning that only a fraction of this signal might be captured during the sensing interval; iii) to consider that the sensed bandwidth is not necessarily equal to the bandwidth of the primary signal and that the center frequencies of the sensed band and the primary signal band might not be the same. All of these new investigations could be carriedout under nonuniform and dynamical noise and signal-tonoise ratios at the secondary user terminals, also considering spatially-correlated shadowing affecting the mobile nodes of the secondary network.

\section{REFERENCES}

[1] R. C. D. V. Bomfin, L. S. Costa, D. A. Guimarães, and R. A. A de Souza, "Original title in Portuguese: Sensoriamento espectral cooperativo sob diferentes intensidades de ruído nos receptores," XXXIV Brazilian Telecommun. Symp., SBrT'16, pp. 979-983, Sep. 2016. [Online]. Available: http://www.sbrt.org.br/sbrt2016/anais/ST32/ 1570277036.pdf

[2] M. A. McHenry, P. A. Tenhula, D. McCloskey, D. A. Roberson, and C. S. Hood, "Chicago spectrum occupancy measurements \& analysis and a long-term studies proposal," in Proc. of the first int. workshop on Technology and policy for accessing spectrum, ser. TAPAS '06. New York, NY, USA: ACM, 2006. doi: 10.1145/1234388.1234389

[3] J. Mitola III and G. Q. Maguire Jr., "Cognitive radio: making software radios more personal,” IEEE Personal Commun. Mag., vol. 6, no. 4, pp. 13-18, Aug. 1999. doi: 10.1109/98.788210

[4] I. F. Akyildiz, B. F. Lo, and R. Balakrishnan, "Cooperative spectrum sensing in cognitive radio networks: A survey," Physical commun., vol. 4, no. 1, pp. 40-62, 2011. [Online]. Available: https://doi.org/10.1016/j.phycom.2010.12.003

[5] H. Urkowitz, "Energy detection of unknown deterministic signals," Proc. of the IEEE, vol. 55, no. 4, pp. 523-531, Apr. 1967. doi: 10.1109/PROC.1967.5573

[6] F. F. Digham, M. S. Alouini, and M. K. Simon, "On the energy detection of unknown signals over fading channels," IEEE Trans. Commun., vol. 55, no. 1, pp. 21 -24, Jan. 2007. doi: 10.1109/TCOMM.2006.887483

[7] T. Yucek and H. Arslan, "A survey of spectrum sensing algorithms for cognitive radio applications," IEEE Commun. Surveys Tuts., vol. 11, no. 1, pp. 116-130, quarter 2009. doi: 10.1109/SURV.2009.090109

[8] W. A. Gardner, "Signal interception: a unifying theoretical framework for feature detection," IEEE Trans. Commun., vol. 36, no. 8, pp. 897906, Aug. 1988. doi: 10.1109/26.3769

[9] Z. Quan, S. Cui, A. H. Sayed, and H. V. Poor, "Optimal multiband joint detection for spectrum sensing in cognitive radio networks," IEEE Trans. Signal Process., vol. 57, no. 3, pp. 1128-1140, Mar. 2009. doi: 10.1109/TSP.2008.2008540

[10] P. Paysarvi-Hoseini and N. C. Beaulieu, "Optimal wideband spectrum sensing framework for cognitive radio systems," IEEE Trans. Signal Process., vol. 59, no. 3, pp. 1170-1182, Mar. 2011. doi: 10.1109/TSP.2010.2096220

[11] Z. Tian and G. B. Giannakis, "A wavelet approach to wideband spectrum sensing for cognitive radios," in Cognitive Radio Oriented Wireless Networks and Commun., 2006. 1st Int. Conf. on, Jun. 2006. doi: 10.1109/CROWNCOM.2006.363459 pp. 1-5.

[12] R. Qiu, N. Guo, H. Li, Z. Wu, V. Chakravarthy, Y. Song, Z. Hu, P. Zhang, and Z. Chen, "A unified multi-functional dynamic spectrum access framework: Tutorial, theory and multi-GHz wideband testbed," Sensors, vol. 9, no. 8, pp. 6530-6603, 2009. doi: 10.3390/s90806530. [Online]. Available: http://www.mdpi.com/1424-8220/9/8/6530

[13] Z. Tian and G. B. Giannakis, "Compressed sensing for wideband cognitive radios," in Acoustics, Speech and Signal Process., 2007. ICASSP 2007. IEEE Int. Conf. on, vol. 4, Apr. 2007. doi: 10.1109/ICASSP.2007.367330 pp. IV-1357-IV-1360.

[14] S. Sedighi, A. Taherpour, S. Gazor, and T. Khattab, "Eigenvalue-based multiple antenna spectrum sensing: Higher order moments," IEEE Trans. Wireless Commun., vol. 16, no. 2, pp. 1168-1184, Feb. 2017. doi: 10.1109/TWC.2016.2640299 
[15] Z. Li, D. Wang, P. Qi, and B. Hao, "Maximum-eigenvalue-based sensing and power recognition for multiantenna cognitive radio system," IEEE Transactions on Vehicular Technology, vol. 65, no. 10, pp. 8218-8229, Oct. 2016. doi: 10.1109/TVT.2015.2511783

[16] M. Arts and R. Mathar, "Performance limits of cooperative eigenvaluebased spectrum sensing under noise calibration uncertainty," in 2016 Eighth Int. Conf. on Ubiquitous and Future Networks (ICUFN), Jul. 2016. doi: 10.1109/ICUFN.2016.7537025 pp. 241-246.

[17] B. Nadler, F. Penna, and R. Garello, "Performance of eigenvaluebased signal detectors with known and unknown noise level," in Commun. (ICC), 2011 IEEE Int. Conf. on, Jun. 2011. doi: 10.1109/icc.2011.5963473 pp. 1-5.

[18] A. Kortun, T. Ratnarajah, M. Sellathurai, C. Zhong, and C. B. Papadias, "On the performance of eigenvalue-based cooperative spectrum sensing for cognitive radio," IEEE J. Sel. Topics Signal Process., vol. 5, no. 1, pp. 49-55, Feb. 2011. doi: 10.1109/JSTSP.2010.2066957

[19] S. Xu, Y. Shang, and H. Wang, "Eigenvalues based spectrum sensing against untrusted users in cognitive radio networks," in Cognitive Radio Oriented Wireless Networks and Commun., 2009. CROWNCOM '09. 4th Int. Conf. on, Jun. 2009. doi: 10.1109/CROWNCOM.2009.5188923 pp. $1-6$.

[20] Y. Zeng and Y.-C. Liang, "Eigenvalue-based spectrum sensing algorithms for cognitive radio," IEEE Trans. Commun., vol. 57, no. 6, pp. 1784-1793, Jun. 2009. doi: 10.1109/TCOMM.2009.06.070402

[21] R. Li, L. Huang, Y. Shi, and H. C. So, "Gerschgorin disk-based robust spectrum sensing for cognitive radio," in 2014 IEEE Int. Conf. on Acoustics, Speech and Signal Process. (ICASSP), May 2014. doi: 10.1109/ICASSP.2014.6855013 pp. 7278-7282.

[22] D. Ramirez, G. Vazquez-Vilar, R. Lopez-Valcarce, J. Via, and I. Santamaria, "Detection of rank- $p$ signals in cognitive radio networks with uncalibrated multiple antennas," IEEE Trans. Signal Process., vol. 59, no. 8, pp. 3764-3774, Aug. 2011. doi: 10.1109/TSP.2011.2146779

[23] L. S. Costa, R. C. D. V. Bomfin, D. A. Guimarães, and R. A. A. de Souza, "Original title in Portuguese: Desempenho de técnicas de fusão para sensoriamento espectral cooperativo sob sombreamento correlacionado," XXXIV Brazilian Telecommun. Symp., SBrT'16, pp. 984-988, Sep. 2016. [Online]. Available: http://www.sbrt.org.br/sbrt2016/anais/ST32/1570277456.pdf

[24] Z. Pourgharehkhan, A. Taherpour, J. Sala-Alvarez, and T. Khattab, "Correlated multiple antennas spectrum sensing under calibration uncertainty," IEEE Wireless Commun. Lett., vol. 14, no. 12, pp. 6777-6791, Dec. 2015. doi: 10.1109/TWC.2015.2459706

[25] R. Gao, Z. Li, P. Qi, and H. Li, "A robust cooperative spectrum sensing method in cognitive radio networks," IEEE Commun. Lett., vol. 18 , no. 11, pp. 1987-1990, Nov. 2014. doi: 10.1109/LCOMM.2014.2361851

[26] R. C. D. V. Bomfin, D. A. Guimarães, and R. A. A. de Souza, "On the probability of false alarm of the power spectral density split cancellation method," IEEE Wireless Commun. Lett., vol. 5, no. 2, pp. 164-167, Apr. 2016. doi: 10.1109/LWC.2015.2514104

[27] R. C. D. V. Bomfin, R. A. A. de Souza, and D. A. Guimarães, "Circular folding cooperative power spectral density split cancellation algorithm for spectrum sensing," IEEE Commun. Lett., vol. 21, no. 2, pp. 250-253, Feb. 2017. doi: 10.1109/LCOMM.2016.2630700

[28] IEEE Standard for information technology- Local and metropolitan area networks-Specific requirements-Part 22: Cognitive Wireless RAN Medium Access Control (MAC) and Physical Layer (PHY) specifications: Policies and procedures for operation in the TV Bands, IEEE Std., Jul. 2011.

[29] H. Kim, J. Kim, S. Yang, M. Hong, M. Yoo, W. Lee, and Y. Shin, "An effective MIMO-OFDM transmission scheme for IEEE 802.22 WRAN systems," in 2007 2nd Int. Conf. on Cognitive Radio Oriented Wireless Networks and Commun., Aug. 2007. doi: 10.1109/CROWNCOM.2007.4549830 pp. 394-399.

[30] S. Atapattu, C. Tellambura, and H. Jiang, "Energy detection based cooperative spectrum sensing in cognitive radio networks," IEEE Trans. Wireless Commun., vol. 10, no. 4, pp. 1232-1241, Apr. 2011. doi: 10.1109/TWC.2011.012411.100611

[31] L. Khalid and A. Anpalagan, "Reliability-based decision fusion scheme for cooperative spectrum sensing," Commun., IET, vol. 8, no. 14, pp. 2423-2432, Sep. 2014. doi: 10.1049/iet-com.2014.0205

[32] A. Singh, M. Bhatnagar, and R. Mallik, "Cooperative spectrum sensing in multiple antenna based cognitive radio network using an improved energy detector," IEEE Commun. Lett., vol. 16, Jan. 2012. doi: 10.1109/lcomm.2011.103111.111884

[33] J. O. Smith, Introduction to Digital Filters with Audio Applications. http://www.w3k.org/books/: W3K Publishing, 2007. ISBN 978$0-9745607-1-7$
[34] I. Lee, J. Son, J. Lee, E. Choi, and S. Lee, "Signal receiving apparatus and method for wireless communication system using multiple antennas," Aug. 19 2014, uS Patent 8,811,537. [Online]. Available: https://www.google.com/patents/US8811537

[35] M. Wax and T. Kailath, "Detection of signals by information theoretic criteria," IEEE Trans. Acoust., Speech, Signal Process., vol. 33, no. 2, pp. 387-392, Apr. 1985. doi: 10.1109/TASSP.1985.1164557

[36] D. A. Guimarães, C. R. N. da Silva, and R. A. A. de Souza, "Cooperative spectrum sensing using eigenvalue fusion for OFDMA and other wideband signals," J. of Sensor and Actuator Networks, vol. 2, no. 1, pp. 1-24, 2013. doi: 10.3390/jsan2010001. [Online]. Available: http://www.mdpi.com/2224-2708/2/1/1/htm

[37] D. A. Guimarães, Digital Transmission: A Simulation-Aided Introduction with VisSim/Comm, 1st ed. Springer Publishing Company, Incorporated, 2009. ISBN 3642013589, 9783642013584

[38] S. Chaudhari, J. Lundén, and V. Koivunen, "On the BEP walls for soft decision based cooperative sensing in cognitive radios," in 2013 IEEE Int. Conf. on Commun. (ICC), Jun. 2013. doi: 10.1109/ICC.2013.6654932 pp. 2627-2632.

[39] S. Chaudhari, J. Lundén, V. Koivunen, and H. V. Poor, "BEP walls for cooperative sensing in cognitive radios using K-out-of-N fusion rules," Signal Process., vol. 93, no. 7, pp. 1900-1908, Jul. 2013. doi: 10.1016/j.sigpro.2013.01.016. [Online]. Available: http://dx.doi.org/10.1016/j.sigpro.2013.01.016

[40] S. Chaudhari, J. Lundén, and V. Koivunen, "BEP walls for cooperative bayesian detection with reporting channel errors," in 2012 IEEE 23rd Int. Symp. on Personal, Indoor and Mobile Radio Commun. - (PIMRC), Sep. 2012. doi: 10.1109/PIMRC.2012.6362714 pp. 2166-2172.

[41] - "BEP walls for collaborative spectrum sensing," in 2011 IEEE Int. Conf. on Acoustics, Speech and Signal Processing (ICASSP), May 2011. doi: 10.1109/ICASSP.2011.5946285 pp. 2984-2987.

[42] L. S. Costa, D. A. Guimarães, and R. A. A. de Souza, "Original title in Portuguese: Desempenho e tráfego sob o efeito da codificação de bloco nas fusões de decisões e de autovalores para sensoriamento espectral de sinais OFDMA," XXXIII Brazilian Telecommun. Symp., SBrT'15, Sep. 2015.

[43] D. A. Guimarães, L. dos Santos Costa, and R. A. A. de Souza, "Comparison between eigenvalue fusion and decision fusion for spectrum sensing of OFDMA signals under errors in the control channel," in 2014 Int. Telecommun. Symp. (ITS), Aug. 2014. doi: 10.1109/ITS.2014.6947981 pp. $1-5$.

[44] L. S. Costa, D. A. Guimarães, and R. A. A. de Souza, "Performance and reporting channel traffic of eigenvalue fusion and block-coded decision fusion for spectrum sensing of OFDMA signals," J. of Commun. and Inf. Syst., vol. 31, Feb. 2016. doi: https://doi.org/10.14209/jcis.2016.4. [Online]. Available: https://jcis.sbrt.org.br/jcis/article/view/375

[45] D. A. Guimarães, L. d. S. Costa, and R. A. A. de Souza, "Performance-traffic tradeoff in eigenvalue fusion and decision fusion for spectrum sensing of OFDMA signals under errors in the reporting channel," Telecommun. Syst., vol. 63, no. 4, pp. 505521, Dec. 2016. doi: 10.1007/s11235-016-0138-6. [Online]. Available: https://doi.org/10.1007/s11235-016-0138-6

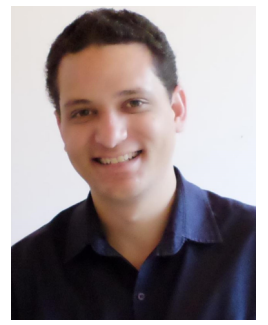

Lucas dos Santos Costa was born in Brazil in 1982. He received the B.S.E.E. and the M.Sc. degrees from the National Institute of Telecommunications, INATEL, in 2011 and 2014, respectively. Since 2015 he has been working at the Reference Center for Radiocommunications, whose research is concentrated on the fifth-generation (5G) of wireless and mobile communications. His main area of interest is related to digital communication systems and, specifically, he has been working with spectrum sensing for cognitive radio applications. 


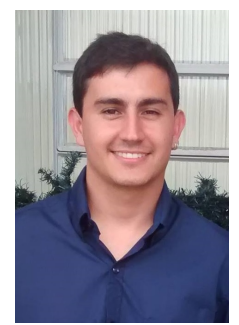

Roberto Cesar Dias Vilela Bomfin received his B.Sc. degree in Electrical Engineering from the National Institute of Telecommunications, INATEL, Brazil, 2014. From 2013 to 2015, he worked as teaching assistant in the undergraduate Digital Communications course of INATEL. In 2014, he worked as a researcher for the Brazilian undergraduate research program, whose topic was to evaluate the wireless channel effects in eigenvalue-based spectrum sensing techniques for cognitive radio networks. In 2016 he received his M.Sc degree in Telecommunications from INATEL, whose contributions were to propose a modification in a existing spectrum sensing technique and deriving some cumulative distribution functions for some random variables produced by the studied detection method. In September of 2016, he joined the Vodafone Chair Mobile Communications Systems at the Technische Universität Dresden (TUD), Germany, as Ph.D. student. His research and interest topics include non-orthogonal 5G waveforms, non-orthogonal multiple access (NOMA), spectrum sensing and statistics.

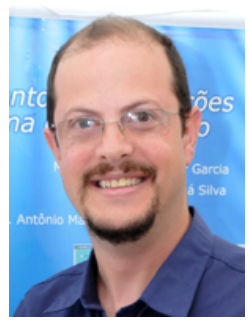

Dayan Adionel Guimarães was born in Carrancas, MG, Brazil, on March 01, 1969. He holds the titles: Electronics Technician (1987), Electrical Engineer (1994), Specialist in Data Communication Engineering (2003), Specialist in Human Resources Management (1996), Master in Electrical Engineering (1998) and Doctor in Electrical Engineering (2003). In 2010 he attended a Pos-Doctoral internship at Federal University of Santa Catarina (UFSC), studying Convex Optimization. From 1988 to 1993 he developed equipment for Industrial Instrumentation and Control, and also occupied the positions of Manufacturing and Product Engineering Supervisor at SENSE Sensors and Instruments. Since January 1995 he is a Professor at the National Institute of Telecommunications, INATEL, where for eight years he was responsible for the structure that supports practical teaching activities for the Electrical Engineering undergraduate course. His research includes the general aspects of fixed and mobile wireless communications, specifically digital transmission, spectrum sensing for cognitive radio, radio propagation, and convex optimization and signal processing applied to communications.

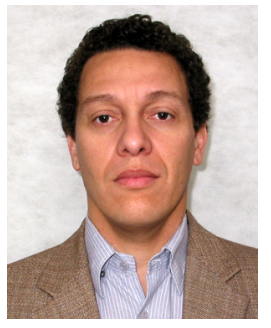

Rausley Adriano Amaral de Souza was born in Brazil in 1972. He received the B.S.E.E. and the M.Sc. degrees from the National Institute of Telecommunications, INATEL, in 1994 and 2002, respectively, and the Ph.D. degree from School of Electrical and Computer Engineering of the State University of Campinas, UNICAMP, Brazil. From 1995 to 2001, he worked as a Purchase Manager at Leucotron Equipamentos Ltda. He joined the National Institute of Telecommunication, INATEL, in 2002, where he is a Full Professor. He is presently a Researcher of the CNPq. Since 2015 he has been working at the Radiocommunication Reference Center, whose researches are concentrated on the fifth-generation (5G) systems. He is a Member of the Brazilian Telecommunications Society (SBrT) and is a Member of the IEEE. His general research interests include wireless communications, fading channel modeling and simulation, diversity systems, and spectrum sensing in cognitive radio systems. 